\title{
Enzyklopädien der Aufklärung in europäischen Vernakularsprachen und der Wissenstransfer über "Modell, Imitation und Kopie“
}

Encyclopédies des Lumières dans les langues vernaculaires européennes et rôle des « modèles, imitations " et « copies » dans les transferts de savoir Encyclopedias of the Enlightenment in vernacular languages and the knowledge transfer via "model, imitation" and "copy"

Ina Ulrike Paul

\section{(2) OpenEdition}

\section{Journals}

Édition électronique

URL : https://journals.openedition.org/ceg/285

DOI : $10.4000 /$ ceg. 285

ISSN : 2605-8359

Éditeur

Presses Universitaires de Provence

Édition imprimée

Date de publication : 5 avril 2017

Pagination : 23-34

ISBN : 979-10-320-0103-5

ISSN : 0751-4239

\section{Référence électronique}

Ina Ulrike Paul, „Enzyklopädien der Aufklärung in europäischen Vernakularsprachen und der

Wissenstransfer über "Modell, Imitation und Kopie"“", Cahiers d'Études Germaniques [Online], 72 | 2017 , Online erschienen am: 05 Oktober 2018, abgerufen am 21 September 2021. URL: http://

journals.openedition.org/ceg/285 ; DOl: https://doi.org/10.4000/ceg.285 


\title{
Enzyklopädien der Aufklärung in europäischen Vernakularsprachen und der Wissenstransfer über „Modell, Imitation und Kopie“
}

Ina Ulrike PAUL

Freie Universität Berlin

\begin{abstract}
The Enlightenment was the first attempt of Europe's Republic of Letters to conduct a cosmopolitan conversation without a 'universal' language. [...] Enlightenment's fundamental ideas - progress, freedom of thought, universal humanity, and critical reasoning - proved highly translatable. Although formidable differentials were on the horizon, Europe's cosmopolitan legacy stood the first test of multilingual modernity. ${ }^{1}$
\end{abstract}

Enzyklopädien, Universallexika und Wörterbücher in den Landessprachen (Vernakularsprachen) Europas zählen zu den zentralen Wissensmedien der Aufklärung. ${ }^{2}$ Von den Enzyklopädien des 16. Jahrhunderts unterschied sie die aufklärerisch-progressive Intention ihrer Wissenssammlung: So vollständig wie möglich sollten die bisher erlangten Wissensbestände der Menschheit zusammengefasst, mit den neuen Ideen und den modernen Denkformen amalgamiert und dann das von beiden revolutionierte Wissen im Medium der

1. Fania 0z-Salzberger, „Translation“, in Alan Charles Kors (Hrsg.), Encyclopedia of the Enlightenment, Bd. 4, Oxford (Oxford University Press) 2003, S. 181-188, 181, 182: “Die Aufklärung war der erste Versuch der Gelehrtenrepublik Europas, eine kosmopolitische Unterhaltung ohne 'universale' Sprache zu führen. ... Die grundlegenden Ideen der Aufklärung Fortschritt, Freiheit des Denkens, allgemeine Humanität und kritische Vernunft - erwiesen sich als in hohem Maße übersetzbar. Obwohl sich beeindruckende Unterschiede am Horizont zeigten, bestand Europas kosmopolitisches Erbe den ersten Test der vielsprachigen Moderne.“

2. Steffen Martus, Aufklärung. Das deutsche 18. Jahrhundert. Ein Epochenbild, Berlin, Rowohlt, 2016; Peter Burke, „Die Explosion des Wissens“. Von der Encyclopédie bis Wikipedia, Berlin,Wagenbach, 2014; Ulrich Johannes Schneider, Die Erfindung des allgemeinen Wissens. Enzyklopädisches Schreiben im Zeitalter der Aufklärung, Berlin, Akademie, 2013; Lumières! Un héritage pour demain, sous la direction de Yann Fauchoir/ Thierry Grillet/Tzvetan Todorov, Paris 2006 ; Silvia Serena Tschopp, Popularisierung gelehrten Wissens im 18. Jahrhundert. Institutionen und Medien, in Richard van Dülmen/ Sina Rauschenbach (Hrsg.), Macht des Wissens. Die Entstehung der modernen Wissensgesellschaft, Köln, Weimar, Wien, Böhlau, 2004, S. 469-489, bes. 471-472. 
Enzyklopädie möglichst Vielen zugänglich gemacht werden. Infolgedessen wurden „les sciences, les arts et les métiers“ der gebildeten Allgemeinheit ohne die traditionellen Hürden humanistischer Gelehrsamkeit präsentiert: Das erste enzyklopädische Lexikon modernen Typus‘ erschien Ende des 17. Jahrhunderts in französischer Sprache als der lingua franca Europas, die die gelehrte Universalsprache Latein ablösen sollte; nur wenige Jahre später lagen auch Enzyklopädien in anderen europäischen Sprachen vor. Zugleich wurde die bisher geltende systematische Darstellung des Wissens durch alphabetisch angeordnete Stichworte (Lemmata) ersetzt, die selbst Laien das gezielte Nachschlagen gesuchter Begriffe ermöglichten. Der neue Lesemodus lemmatisierter lexikalischer Werke war der noch heute gültige für enzyklopädische Werke, nämlich der der partiellen, konsultierenden Lektüre. Schließlich sollten die Enzyklopädien der Aufklärung „utiles et nécessaires, aux Amateurs des Sciences \& des Beaux Arts, Et principalement à ceux qui désirent d'entrer dans le commerce du monde, Et de pouvoir fournir à la conversation des honnêtes gens " sein. ${ }^{3}$ Die Zielgruppe des Mediums Enzyklopädie im ersten halben Jahrhundert der Aufklärung waren demnach „die Wohlerzogenen, Belesenen und Weitgereisten“. Verleger, Herausgeber und Autoren suchten anfangs nach einem gemeinsamen anspruchsvollen Sprachstil für Gelehrte und Gebildete. Im Lauf der Zeit richteten sie ihre Enzyklopädien stilistisch-inhaltlich stärker auf jene an der Aufklärung interessierten Leserinnen und Leser aus, die auch populär(wissenschaftlich)e Werke der Literatur, Philosophie, Historiographie oder Ethnographie im Original lesen konnten, sie aber lieber in der eigenen Landessprache lasen. Zugunsten dieses Lesepublikums hatten Universal- wie Spezialenzyklopädien in ihrer Eigenschaft als neue Medien der Wissensvermittlung und -vermehrung zugunsten Vieler auf ihre Weise Anteil an der Weitergabe von „unterhaltsame[m] Wissen“, wie es sich bei beispielsweise bei ethnographischen Stichworten zum „caractère des nations“ oder „Naturell der Völcker“ oder in politisch-geographischen Artikeln wie zu Europa nachlesen lässt: ${ }^{5}$

On dit que les François sont polis, adroits, généreux, mais prompts \& inconstan[t]s; les Allemands, sincères, laborieux, mais pesan[t]s, \&t trop adonnés au vin; les Italiens agréables, fins, doux en leur langage, mais jaloux \& traîtres; les Espagnols secrets, pruden[t]s, mais rodomonts, \&t trop formalistes; les Anglois orgueilleux, méprisans \&t fiers jusqu'à la férocité ${ }^{\text {. }}$

Selbst der große Aufklärer Pierre Bayle sah sich verpflichtet, dem Unterhaltungsbedürfnis des Publikums in seinem Dictionnaire historique et

3. Samuel Chappuzeau, Dessein d'un Nouveau Dictionaire Historique, Géographique, Chronologique Et Philosophique, Celle, André Holwein, 1694, Titelblatt.

4. „[T]he well-bred, well-read and well-travelled“, in Oz-Sandberger, "Translation”, S. 181.

5. Markus Fauser, Wissen als Unterhaltung, in: Richard van Dülmen / Sina Rauschenbach (Hrsg.), Macht des Wissens, S. 491-514, Zitat: 496; Ingrid Tomkowiak (Hrsg.), Populäre Enzyklopädien. Von der Auswahl, Ordnung und Vermittlung des Wissens, Zürich, Chronos, 2002, S. 197-221.

6. Art. Europe, in Louis Moréri, Le Grand Dictionnaire Historique, 2. Aufl. 1681 - 20. Aufl. Paris 1759; Ina Ulrike Paul, „Wache auf und lies...“. Zur Tradierung von Nationalstereotypen in europäischen Enzyklopädien des 18. Jahrhunderts, in Tomkowiak, Populäre Enzyklopädien, S. 197-221. 
critique mit Einschüben Rechnung zu tragen, für deren Obszönität er sich in der zweiten Auflage zu rechtfertigen hatte. ${ }^{7}$

Wer unter der inspirierenden Thematik „Modèle, imitation, copie“8 über den Wissenstransfer in den enzyklopädischen Universallexika des Zeitalters der Aufklärung nachdenkt ${ }^{9}$, müsste sich - könnte Vollständigkeit hier das Ziel sein - jeweils mit den ,Marksteinen“ unter den Enzyklopädien in zehn Landessprachen beschäftigen, die bis zum Ende des 18. Jahrhunderts zwischen Lissabon und St. Petersburg, Venedig und Stockholm erschienen waren, und die Frage zu beantworten suchen, welche Werke denn zu „Modellen“ für die nachfolgenden geworden seien? Obwohl diese Frage im weiteren Verlauf am Beispiel der allerersten dieser modernen Enzyklopädien erörtert wird, seien die beiden bekanntesten Enzyklopädien der Aufklärung und zugleich die wichtigsten Modelle für die Weiterentwicklung der Gattung zumindest erwähnt, nämlich die Cyclopaedia (1728) von Ephraim Chambers und die an ihr als Modell orientierte Encyclopédie, ou Dictionnaire raisonné des Sciences, des Arts et des Métiers (1751-1772) von Denis Diderot und Jean le Rond d'Alembert - beide verdanken ihre Entstehung dem Wunsch, ein vorhandenes Modell verbessert nachzubilden.

Der im Anschluss an den Komplex „Modell“ zu besprechende Schlüsselbegriff „Imitation“ veranlasst zu der Frage, welche enzyklopädischen Werke sich gegenüber dem eruierten Modell (oder mehreren Modellen) als Imitationen erweisen? Im Gegensatz zu der passiven Funktion eines „Modells“ ist die der „Imitation“ ein aktiver Akt. Kann man aber eine Enzyklopädie - die im 18. Jahrhundert als ein mit Druckprivileg ausgestattetes, mehrbändiges, ledergebundenes Werk in Folio vorzustellen ist - überhaupt „imitieren“ im Sinne des Nachbildens, Nachahmens, Fälschens oder Vortäuschens?

Der letzte Schlüsselbegriff „Kopie“, also Vervielfältigung und Replikation müsste er nicht an zweiter Stelle stehen? Sind die in ihrer äußerlichen Gestalt (denken wir wieder an das Folioformat, an den Titel oder die teure Ausstattung mit Kupferstichen, Vignetten und Abbildungen) und/oder in ihrem inneren Gehalt (Systematik, Wissensgehalt) ,kopierten“ enzyklopädischen Werke nicht näher am Modell als die nur ,imitierten‘? So soll die dritte Frage zur Anfangsprämisse

7. Günter Gawlick/Lothar Kreimendahl (Hrsg. und Übers.), Pierre Bayle, Historisches und kritisches Wörterbuch. Eine Auswahl, Hamburg, Felix Meiner, 2003, S. XXIII.

8. Zur Definition der drei Begriffe s. Christina Stange-Fayos/ Katja Wimmer, Journée d'Etudes "Modèle, Imitation, Copie ". Organisée dans le cadre de la nouvelle thématique du CREG EA 4151 Héritages et transmission. Université Paul Valéry, Montpellier les jeudi 26 et vendredi 27 mars 2015 (CfP 2014).

9. Claudine Cohen, Sciences (Diffusion et vulgarisation de), in: Michel Delon (Hrsg.), Dictionnaire européen des lumières, Paris (Presses Universitaires de France) 1997, S. 979-981, bes. 981 (die Popularisierung und der Transfer des Wissens durch Universallexika wird allerdings erst mit der Encyclopédie angesetzt); Martin Schierbaum, Typen von Transformationen der Wissensspeicher in der Frühen Neuzeit - zwischen Marktmacht, Praxisdruck und suisuffizienter Welterklärung. [...], in Ders. (Hrsg.), Enzyklopädistik 1550-1650. Typen und Transformationen von Wissensspeichern und Medialisierungen des Wissens (= Pluralisierung \& Autorität Bd. 18), Berlin, (Litt), 2009. 
zurückführen und erörtern, wo und wie die Dreiheit „Modell“, „Imitation“ und „Kopie“ den Transfer von Wissen befördert.

\section{Modell oder Modelle?}

In der Formierungsphase der modernen Wissenschaften wurden bahnbrechende Philosophen wie René Descartes und John Locke sowohl in ihren Landessprachen als auch in Übersetzungen gelesen. Autorisiert waren solche Übersetzungen damals meist nicht, doch schadeten sie dem Original weniger als sie seiner Verbreitung nützten. Die ,lateinischen“ Autoren Baruch de Spinoza, Samuel Pufendorf oder Isaac Newton errangen ihre herausragende Bedeutung für die Aufklärung vornehmlich durch die Übersetzungen ihrer Werke in das Französische. ${ }^{10}$ Vor diesem Hintergrund lag es nahe, das erste nichtlateinische und aus alphabetisierten Lemmata bestehende Universallexikon für die gebildeten Milieus in französischer Sprache erscheinen zu lassen. Als Werk nur eines einzigen Gelehrten, des aus dem südfranzösischen Bargemont stammenden Abbé Louis Moréri S.J. (1643-1680), wurde Le Grand Dictionnaire Historique 1674 in Lyon gedruckt und stieß auf Anhieb auf größtes Interesse der frankophonen Leserschaft, wie die rasch aufeinander folgenden und an Zahl der Bände fortwährend zunehmenden Auflagen belegen: acht waren es allein bis zum Jahrhundertwechsel, und bis 1759 sollte ,der Moréri' noch zwölf weitere französischsprachige Editionen erleben - ganz zu schweigen von den englischen, deutschen, niederländischen, italienischen und spanischen Übersetzungen. ${ }^{11}$ Abbé Moréri, dieser erste, wenn auch nicht mit ihnen vergleichbare Vorläufer der Enzyklopädisten, starb ein Jahr vor der zweiten Auflage seines Werkes von 1681. Der in deren Vorwort ausgeführte verlegerische Wunschtraum, mit einem enzyklopädischen Universallexikon die unterschiedlichen Gruppen der Gelehrten und spezialisierten Wissenschaftler, der Gebildeten und weltläufigen Besucher von Salons gleichermaßen anzusprechen, erfüllte sich weit über alle Hoffnungen. ${ }^{12}$

10. Abgewandelt zitiert und übersetzt nach: Oz-Sandberger (Anm. 1), S. 181. Zu den Pufendorf -Übersetzungen s. Martus, Aufklärung, S. 74.

11. $\mathrm{Zu}$ den Übersetzungen s. Arnold Miller, Louis Moréri's Grand dictionnaire historique, in Frank A. Kafker (Hrsg.), Notable encyclopedias of the seventeenth and eighteenth centuries: nine predecessors of the Encyclopédie, Oxford, Voltaire Foundation, 1981, S. 13-47; Gert A. Zischka, Index Lexicorum: Bibliographie der Lexikalischen Nachschlagewerke, Wien 1959, S. 7. - Folgende Ausgaben wurden für diesen Aufsatz herangezogen: [Louis Moréri], Le Grand Dictionnaire Historique ou Le Mélange Curieux de l'Histoire Sacrée et Profane [...], 2nde éd. revue, corrigée et augmentée, 2 vol. Lyon 1681 et 1 suppl.; 5ième éd. [...] 3 vols. (2 vol., 1 suppl.) Lyon, Paris 1688 ; 9ième éd. [...] 4 vol. Amsterdam, La Haye 1702 ; 11ième éd. [...] 4 vol. Amsterdam, La Haye, Utrecht 1724 ; 20ième éd. [...] 10 vol., éd. par [Étienne François] Drouet. Paris 1759.

12. Ab der 2. Aufl. des Moréri von 1681, die 1689 durch ein Supplement ergänzt wurde, wurde im Vorwort klar die Zielgruppe der Enzyklopädie angesprochen: die Gelehrten („Les doctes“), die Gebildeten (,à ceux qui désirent de sçavoir un peu de tout“), die Wissenschaftler (,aux Sçavans pour se rafraîchir la mémoire“) und schließlich noch diejenigen Leserinnen und Leser an, die das 
Obwohl Moréris französischsprachiges Werk europaweit gelesen werden konnte, erschienen mit der Jahrhundertwende bereits Übersetzungen ins Englische und Deutsche - ein deutliches Zeichen für den Modell-Charakter des französischen Werkes, das dennoch die spezifischen Erwartungen des Lesepublikums dieser Länder nicht hatte erfüllen können. Ungeteilte Zustimmung erhielt Le Grand Dictionnaire ohnehin nicht; vielmehr zog es aufgrund seiner vielen sachlichen Fehler und seiner dezidiert katholischen Weltsicht weit über Frankreich hinaus wissenschaftlich gerechtfertigte und dem aufgeklärt-religionskritischen Zeitgeist verpflichtete Kritik auf sich. Der bedeutendste seiner Kritiker war der nach der Aufhebung des Ediktes von Nantes in die Niederlande emigrierte Philosoph und Schriftsteller Pierre Bayle. Eigentlich mit einem um die gleiche Leserschicht konkurrierenden Werk beauftragt, setzte Bayle Moréris katholisch-orthodoxem Dictionnaire eine Abrechnung mit religiösem Dogmatismus, Idolatrie und Wahnglauben entgegen: sein Dictionnaire historique et critique (1695/96) war ein Aufklärungsmanifest, wie es später Chambers mit seiner Cyclopedia oder Diderot und D'Alembert mit ihrer Encyclopédie vorlegten. Es wurde in kurzer Zeit trotz seines hohen Preises zu einem glänzenden Buchhandelserfolg und bald zum „philosophischen Klassiker“. ${ }^{13}$ Bayle hatte sich keineswegs auf die Revision des Moréri beschränkt. Seine neuen Lexikonartikel fasste er kurz und ergänzte sie mit langen Fußnoten, in denen er Quellen und Literatur zu einander widersprechenden Meinungen anführte. Diese bahnbrechende Neuerung zwang die Leser dazu, an ihrem eigenen Wissen zu zweifeln und sich zwischen den verschiedenen, teilweise unvereinbaren Positionen der Wissenschaftler zu entscheiden. Kein um Vollständigkeit bemühtes, sondern ein zu unabhängigem Denken aufforderndes Wörterbuch hatte Bayle geschaffen - Kant ein Jahrhundert vorauseilend forderte er die Leserschaft zum sapere aude und darüber hinaus auch zum diiudicare aude des selbständig denkenden Menschen auf. ${ }^{14}$

Ein im Gegensatz zu Bayle bis heute weitgehend unbekannter Moréri-Kritiker brachte seine Missbilligung dieses Modells aller folgenden Enzyklopädien auf bemerkenswerte Weise zum Ausdruck. Der aus dem Poitou stammende, in Genf ansässige hugenottische Lexikograph Samuel Chappuzeau (1625-1701) behauptete nämlich vierzehn Jahre nach Moréris Tod, letzterer hätte sein bis zum Buchstaben „P“ abgeschlossenes Manuskript plagiiert, in dessen Besitz er

Werk läsen, um später „dans les belles Conversations ... sur une infinité de sujets“ mithalten zu können.

13. Pierre Bayle, Dictionnaire historique et critique, 2 Bde, Rotterdam, 1697, wobei hier vorzüglich, die eingeleitete deutsche Ausgabe herangezogen wurde: Günter Gawlick/Lothar Kreimendahl (Hrsg. und Übers.), Pierre Bayle, Historisches und kritisches Wörterbuch. Eine Auswahl, Hamburg, Felix Meiner, 2003, S. IX. Zur Entstehungsgeschichte von Bayles Dictionnaire als Auftragswerk des niederländischen Verlegers Reinier Leers (1654-1714) ebda., XXIII. Vgl. Robert Collison, Encyclopaedias: their history throughout the ages : a bibliographical guide with extensive historical notes to the general encyclopaedias issued throughout the world from 350 B.C. to the present day, New York, Hafner, 1964, p. 88-89.

14. Ernst Cassirer, Die Philosophie der Aufklärung. Text und Anmerkungen bearb. v. Klaus Rosenkranz, mit einer Einleitung v. Gerald Hartung, Hamburg, Felix Meiner, 2007, S. 169. 
unrechtmäßig gekommen sei, und verdanke seinen Erfolg also eigentlich ihm. ${ }^{15}$ Zugleich kündigte Chappuzeau in seinem Dessein d'un nouveau dictionnaire historique ein dreibändiges enzyklopädisches Werk an, das die protestantische Weltsicht in den Wissenschaften spiegeln und damit die tätige Unterstützung der protestantischen Fürsten Europas erringen sollte; es kam jedoch nie zustande. ${ }^{16}$

Die Frage nach dem Modellcharakter der ersten französischsprachigen Enzyklopädie von Louis Moréri für seine Zeitgenossen ist also leicht zu beantworten, obwohl viele überarbeitete Auflagen und übersetzte, avant la lettre nationale Adaptationen folgten: Le Grand Dictionnaire besaß die ModellFunktion sowohl für das berühmte Dictionnaire historique et critique von Pierre Bayle als auch den Enzyklopädie-Plan von Chappuzeau. Letzterer reklamierte nicht nur Idee und Erfolg Moréris, sondern verwandte dessen Werk auch als (Gegen-)Modell für sein religiös-weltanschaulich diametral entgegengesetztes Publikationsprojekt. Für den ungleich bedeutenderen Aufklärer Bayle diente Le Grand Dictionnaire als negatives Modell, das ihm nicht als Grundlage einer Revision, sondern für eine bahnbrechende Neuschöpfung diente.

Während von den folgenden französischen Moréri-Ausgaben die revidierte sechste, von dem Calvinisten Jean Le Clerc herausgegebene neuerdings eigenständigen Modell-Charakter errang, reklamierten die erste deutsche und die erste niederländische Moréri-Übersetzung ihren außergewöhnlichen Stellenwert für die Leserschaft ihres eigenen Landes durch drei Kompetenzen - durch ihre Modelle (denn sie verwiesen nicht nur auf eines), durch ihre Publikation in der Landessprache ihres Lesepublikums und durch die spezifische, religionskritische und ,nationale‘ Adaptation ihres Wissensbestandes.

15. Samuel Chappuzeau (1625-1701), der Erzieher des englischen Königs William III. (1650-1702) und späterer Pagenhofmeister des Herzogs Georg Wilhelm von Braunschweig-Lüneburg (1624-1705) war, wurde zu seiner Zeit immerhin von Pierre Bayle (1647-1706), später von dem Leipziger Lexikographen Johann Franz Budde(us) (1667-1729) und von Johann Heinrich Zedler (Bd. 24) erwähnt. Vgl. Ina Ulrike Paul, „Dieses Universallexicon hat seines Gleichen nicht“ - oder doch? Zedlers enzyklopädische Vorläufer in Europa, in Kai Lohsträter /Flemming Schock (Hrsg.). "Die gesammelte Welt". Wissensformen und Wissenswandel in Zedlers Universal-Lexicon, (= Schriften und Zeugnisse zur Buchgeschichte Bd. 19), Wiesbaden, Harrassowitz, 2013, S. 9-16, S. 24f. Zwei kenntnisreiche biographische Artikel finden sich in der französischen und deutschen Wikipedia: [https://fr.wikipedia.org/wiki/Samuel_Chappuzeau] und [https://de.wikipedia.org/ wiki/Samuel_Chappuzeau] (beide Zugriffe: 20.07.2016).

16. Samuel Chappuzeau, Dessein D'un Nouveau Dictionnaire Historique, Géographie, Chronologique \& Philosophique, qui contient en abbrégé, les Vies des Empereurs, des Rois, des princes illustres At des grans [!] Capitaines: Des Philosophes, des Auteurs anciens \& modernes, des Inventeurs des Arts, \& des autres personnes de toutes sortes de professions, \& de tout sexe, qui se sont distinguées par leur Ecrits, ou par un mérite éclatant: Celles Des Patriarches, des Juges \&t des souverains Pontifes. A Cell (chez André Holwein) MDCXCIV [= 1694] /[deutsch :] Entwurff Eines Neuen Historisch= Geographisch= Chronologisch= und Philologischen Dictionarii / [lateinisch :] Meditatio Novi Dictionarii Historici, Geographici, Cronologici \& Philologici. 


\section{Imitation - welcher Modelle?}

Zunächst einmal ist eine Übersetzung eines enzyklopädischen Werkes keine Imitation ihrer Vorlage, sondern eine Übersetzung. Was aber kann ein Verleger oder Verlagsbuchhändler mittels einer Übersetzung imitieren? Imitiert wurde natürlich das Projekt als solches. Ginge es dabei nur um Äußerlichkeiten, so kann man von Imitation sprechen, weil sich das Format der französisch- und der deutschsprachigen Bände und das Papier ähneln, auch das Vorhandensein der Paratexte (Vorworte, Widmungen etc.) und die Ausstattung mit Kupferstichen und Vignetten, diese selbst jedoch nicht. Der Haupttitel des ersten deutschsprachigen alphabetisierten Universallexikons, das im Jahr 1709 in Leipzig im Verlag von Thomas Fritsch erschien und das an dem Modell Le Grand Dictionnaire Historique ausgerichtet war, lautete denn auch abweichend Allgemeines Historisches Lexicon $^{17}$.

In dessen Vorwort erläuterte der Herausgeber Johann Franz Budde(us), der als Theologe und Orientalist Ansehen in der république des lettres genoss ${ }^{18}$, dass das von ihm vorgelegte enzyklopädische Lexikon auf fünf Werken beruhe, nämlich 1.) auf den drei jüngsten Moréri-Überarbeitungen aus Frankreich (1702), England (1694) und den Niederlanden (1696), 2.) auf Bayles Dictionnaire historique et critique sowie 3.) auf dem 1697 von Antoine Galland fertiggestellten und in einer populärwissenschaftlichen Ausgabe weit bekannten Lebenswerk des Orientalisten Barthélemy d'Herbelot de Molainville mit dem Titel Bibliothèque orientale ou Dictionnaire universel contenant tout ce qui regarde la Connoissance des peuples de l'Orient. $^{19}$ Die Kompilation eines neuen Buches aus anderen Werken galt nicht als Teilplagiat und war auch nicht ehrenrührig, sondern durch die ausdrückliche Nennung der Vorgängerwerke sogar guter wissenschaftlicher Stil - eine Verneigung vor den vorbildhaften Modellen. Letztere demonstriert, dass enzyklopädische Werke wie Lehrbücher auf die vollständige Darbietung von traditionell gesichertem Wissen zielten; erst die Enzyklopädisten um Diderot sollten sich die aufklärerische Revolution der Weltsicht durch die Revolutionierung des Wissens auf die Fahnen schreiben.

17. Allgemeines Historisches Lexicon mit Johann Franz Buddeus“ Vorrede (zitiert als „Leipziger Lexikon“), 4 Bände, 1. Aufl. Leipzig, Thomas Fritsch, 1709; die 2. Auflage erschien 1722, die dritte 1730-32.

18. Der lutherische Theologe und Philosoph Johann Franz Buddeus (1667-1729) lehrte in Halle und Jena.

19. Die neunte, vierbändige Ausgabe des Moréri, Le Grand Dictionaire Historique (Amsterdam, La Haye 1702) bezieht explizit Bayles Kritik in die Neuauflage ein: "Neuvième édition où l'on a mis le supplément dans le même ordre alphabétique, corrigé les fautes censure [sic] dans le Dictionnaire critique de Mr. Bayle \&t grand nombre d'autres \&t ajouté plus de 600 articles et remarques." - Die drei genannten Moréri-Auflagen genossen einen viel besseren Ruf als die Erstauflage: in der 9. Auflage waren viele der von Bayle inkriminierten Fehler Moréris berichtigt worden, die in den Niederlanden publizierte „holländische“ Edition wies die Korrekturen Jean Le Clercs auf und die englischen Ausgabe diente als Grundlage für die neuen Lemmata zu England, Schottland und Irland, die sich in den vorangegangenen französischen Moréri-Editionen nicht fanden. 
Demgegenüber wollte der Leipziger Verleger Fritsch den Verkaufserfolg von Moréris Lexikon im deutschsprachigen Reichsgebiet wiederholen, ohne ein neu(geschaffen)es und allein durch die Vielzahl der notwendigen Mitwirkenden kostspieliges Werk in Auftrag zu geben. Nota bene war der Verleger Leers bei der Anwerbung von Bayle ebenso vorgegangen, und selbst Chambers und Diderot begannen ihre großen Werke als verlegerische Auftragsarbeiten, die an den jeweils gerade marktbeherrschenden großen Enzyklopädien orientiert waren. Im Falle des nach seinem Druckort so genannten „Leipziger Lexikons“ bildeten Übersetzungen aus den zitierten Vorgängerwerken den Hauptteil der Lemmata. Diese alle waren nicht verändert, aber an Bayles Fehlerlisten seiner Moréri-Revision überprüft worden. Der Wissens- und Forschungsstand dieser deutschen Übersetzung war also bis hierher der einer revidierten und übersetzten Neuausgabe der anerkanntesten Moréri-Ausgaben. Herausgeber und Verleger versicherten aber, dass es an „die 2000 gantz neue / und zwar wichtige haupt=articul in unserm Lexico zu finden [gäbe]/ welche weder in Moreri / noch im Bayle, noch in einigen andern Lexico historico anzutreffen" seien. ${ }^{20}$ Hier also waren nicht nur die Lemmata gemeint, die sich Budde als Orientalist schuldig war, denn dem Argument der Neufassung von wesentlichen Lexikonartikeln folgte ein zweites gewichtigeres, das die Bedeutung der Enzyklopädie für den deutschsprachigen Buchmarkt betonen und ihr Leser gewinnen sollte: Dieses Universallexikon, so unterstrich der Herausgeber, sei „hauptsächlich auf den zustand von Teutschland gerichtet“ und käme auf diese Weise besonders denjenigen zustatten, die „in Teutschland leb[t]en / oder auch von den sachen in Teutschland gründlichere nachricht verlang[t]en." Dieser erste Appell an das politisch-kulturelle Identitätsgefühl der Leserschaft einer bestimmten Nationalität und ihr ausgeprägtes Interesse am eigenen Land erwies sich als so erfolgreich, dass spätere Moréri-Übersetzungen in andere Landessprachen ihn regelmäßig für ihre Nation aufnahmen. ${ }^{21}$

So hieß es auch in der seit 1724 erscheinenden niederländischen MorériAdaptation mit dem Titel Het Algemeen Historisch, Geographisch en Genealogisch Woordenboek [...] (Das allgemeine, historische, geographische und genealogische Wörterbuch), dass alle in ihm enthaltenen Artikel über die Niederlande neu verfasst worden und in keinem der zitierten Werke zu finden seien. ${ }^{22}$ Dieses erste Universallexikon in niederländischer Sprache war nach Aussage des 31jährigen Rechtsgelehrten, Historikers und Diplomaten Abraham George Luïscius ein Ein-

20. Leipziger Lexikon, Vorwort des Herausgebers, unpaginiert (eigene Zählung: S. 6).

21. Nach der deutschen folgte die schweizerische Moréri-Adaptation (Anm. 27), dann die genannte niederländische und später diejenigen Spaniens, Italiens, Polens und Schwedens. S. dazu: Paul, „Dieses Universallexicon [...]“, S. 34.

22. Abraham George Luïscius, Het Algemeen Historisch, Geographisch en Genealogisch Woordenboek [...], Alles na de orde van het Alfabet uit de allerbeste Woordenboeken van andere Natien zamengesteld, en met een grote menigte van Artikels, voornamelyk ten opzicht van de zaken van deze Nederlanden, in gene andere Woordenbooken te vinden [...], 8 Bde. 't Gravenhage, Pieter Husson, Delft, Reinier Boitet, 1724-1737, Titelblatt. 
Mann-Unternehmen wie dasjenige Moréris. ${ }^{23}$ Möglicherweise honorierte er aus eigener Tasche Mitarbeiter, wie es etwa von Louis de Jaucourt als Herausgeber der Encyclopédie bekannt ist; vielleicht nahm er die Übersetzung und Überarbeitung für acht dicke Foliobände aber auch alleine auf sich. Die Fertigstellung des Lexikons zog sich jedenfalls über dreizehn Jahre hin und ruinierte Luïscius nicht nur finanziell.

Zu dieser tragischen Entwicklung mag die ein Jahr nach Luïscius‘ Unternehmen auf den Markt geworfene zweite niederländische Enzyklopädie beigetragen haben, das den Titel Groot Algemeen Historisch, Geographisch, Genealogisch, en Oordeelkundig Woordenboek (Großes allgemeines, historisches, geographisches, genealogisches und urteilsfähiges Wörterbuch) trägt. Mit dem fast identischen Titel „Großes Allgemeines Wörterbuch“ anstelle von „Allgemeines Wörterbuch“ konkurrierte es ab 1725 mit der von ihr vollständig ignorierten ersten niederländischen Enzyklopädie von Luïscius und hatte dafür, die besseren Karten: ${ }^{24}$ Zwei über die Universitätsöffentlichkeit hinaus bekannte und höchst produktive niederländische Gelehrte figurierten als Verfasser, während ein Konsortium aus vier Verlagsbuchhändlern die Herausgeberschaft übernommen hatte; zusätzlich war eine große, zum Teil im Vorwort namentlich genannte Mitarbeiterschar für die Zuarbeit sowie die Bearbeitung von Spezialgebieten zuständig. Doch sei hier insbesondere das Frontispiz hervorgehoben. Dieses illustriert nämlich die Aussage der eine ganze Folioseite einnehmenden barocken Titelei, dass das neue Werk auf drei berühmten Vorläufern beruhe. So senkt die allegorische Personifikation der aufgeklärten Wissenschaften eben ihre Schreibfeder, mit der sie einen Augenblick vorher die Namen Moréri, Bayle und Budde(us) unter den Buchtitel gesetzt hatte. In der Widmung betonte das wortführende Buchhändler-Konsortium, dass das hier vorgelegte neue enzyklopädische Werk

23. Der rechtsgelehrte Lexikograph Abraham George Luïscius (* 1693 in Xanten, † nach 1740) wurde am 24. September 1703 in das Universitätsregister von Duisburg eingeschrieben. Vermutlich studierte er auch an der Universität Leyden. Eine niederländische Übersetzung der anonym publizierten „The history of the most serene house of Brunswick-Lunenburgh“ von David Jones ließ Luïscius unter dem Titel Historie des doorluchtigste Huize van Brunswyk-Lunenburg [...]. Uit het Engelsch vertaald, uitgebreid en vermeerdert door A.G. Luiscius 1716 in Amsterdam erscheinen. Seit dem 24. Juni 1734 Auswärtiges Mitglied der Königlich-Preußischen Sozietät [i.e. Akademie] der Wissenschaften in Berlin, war er 1732-1739 und 1742/43 preußischer Resident und außerordentlicher Gesandter bei den Generalstaaten im Haag und zugleich Königlich-Preußischen Domänenrat. 1739 wurde er wegen Unterschlagung abberufen, unternahm einen Selbstmordversuch und verlor alle Ämter. [http://www.bbaw.de/die-akademie/ akademiegeschichte/mitglieder-historisch] (letzter Zugriff: 18.08.2016); Abraham Jacob van der Aa, Biographisch Woordenboek der Nederlanden, Deel 11, Harlem 1865, S. 718-719: [http://www. dbnl.org/tekst/aa_001biog13_01/aa_001biog13_01_1512.php\#11496] (Zugriff: 18.08.2016).

24. David van Hoogstraten, Matthæus Brouërius van Nidek, Jan Lodewyk Schuër, Groot Algemeen Historisch, Geographisch, Genealogisch, en Oordeelkundig Woordenboek [...], 10 Teile Amsterdam u.a. 1725-1733. - Der erste Band des Groot Algemeen Woordenboek erschien 1725, als sein Initiator, der eigentlich als Mediziner ausgebildete Dichter und Literat David van Hoogstraten (1658-1724) bereits verstorben war. Der zweite Herausgeber, der Jurist und Dichter Matthæus Brouërius van Nidek (1677-1742), schied nach dem zweiten Band als Herausgeber aus, um dem historisch gelehrten Bearbeiter, Übersetzer und Kompilator Jan Lodewijk Schuer (1698-nach 1740) unter seiner Aufsicht die Herausgeberschaft zu überlassen. 
durch die Hinzuziehung „unsere[r] eigenen [i.e. niederländischen] Gelehrten“ die „fremdländischen“ Vorgängerwerke als Ausgangsbasis herangezogen, sie aber im Hinblick auf niederländische Belange weit übertroffen habe: „Wir zweifeln nicht“, so ließen sie sich vernehmen, „dass dieses Werk unseren Land- und Sprachgenossen angenehm und nützlich“ sein werde. ${ }^{25}$

Ähnlich wie vorher bei Budde und Luïscius finden wir hier die erwähnte patriotische Standardformulierung für neu lancierte landessprachliche Enzyklopädien. Wenn die Buchhändler ihrer Leserschaft zusätzlich in der langen Vorrede zum ersten Band auseinandersetzten, dass die in Frankreich und den Niederlanden, in England und Deutschland entstandenen Moréri-Übersetzungen und -Bearbeitungen dem Original neuen Glanz verliehen hätten, wenn sie dann zusätzlich auf das „vorzügliche“ (overheerlyk) Wörterbuch des großen Pierre Bayle und die von Jacques Bernard in Amsterdam herausgegebenen Ergänzungsbände des Moréri als Quellen für das eigene Werk hinwiesen, so beschrieben sie damit auch den Prozess des Wissenstransfers durch Übersetzung, Überarbeitung, Zusammenführung mit vergleichbaren Werken und Spezialliteratur als Gewinn für das Ausgangswerk, nicht als Revision von veraltetem und veraltendem Wissen. ${ }^{26}$

\section{Kopie - Nachdruck, Raubdruck?}

Der Verleger des ersten Schweizer Universallexikons mit dem Titel Neu=vermehrtes, Historisch= und Geographisches Allgemeines Lexicon, das 1726 in Basel erschien, warb bei seinen eidgenössischen Landsleuten auf der Titelseite mit inzwischen bekannt klingenden Argumenten für das von ihm verlegte enzyklopädische Werk, die ihr Interesse wecken und seinen Umsatz fördern sollten. ${ }^{27}$ Nicht nur habe der bekannte Basler Theologe Jakob Christoph Iselin, Mitglied der Académie française, das Vorwort verfasst, sondern dieses neue Universallexikon basiere neben allen älteren, von gleichen Materien handelnden Lexika auch auf „bewährter“ historisch-geographischer Spezialliteratur. Alles Material sei gründlich revidiert „und sonderlich was die Schweitzerische und

25. De Compagnie Boekverkopers, Uitgevers van dit Woordenboek (Amsterdam, den 15 October 1725): Opdragt aan den edelen grootachtbaren Here Mr. Jan Trip, Here van Berkenrode [...], in ibid. vol. 1 (1725), 6 p. (unpaginiert). - Zitat: ibid. (eigene Zählung: S. 4) - Jan Trip van Berckenrode (1664-1732) war bis 1727 Regent von Amsterdam.

26. Jacques Bernard, Supplément Aux Anciennes Editions Du Grand Dictionnaire Historique De Mre. Louis Moreri [...], 2 vol. Amsterdam (Pierre Brunel, R. \&t G. Wetstein, David Mortier, Pierre De Coup), La Haye (Adrian Moetjens, L.\&t H. Van Dole), Utrecht (Guillaume van de Water) 1716.

27. Neu vermehrtes Historisch- und Geographisches Allgemeines Lexicon [...].Aus allen vorhin ausgegebenen und von gleichen Materien handlenden Lexicis, auch andern bewährten Historisch= und Geographischen Schrifften zusammen gezogen/ Dißmahlen von neuem mit Fleiß gantz übersehen/von einer grossen Anzahl Fehlern/ die noch immer in denen alten Ausgaben geblieben waren/gereiniget/ und sonderlich was die Schweitzerische und angräntzender Orten und Ländern Sachen betrifft/ganz umgegossen/ und um ein grosses vermehret. Mehr Bericht von allem ist zu finden in der Vorrede/ von Jacob Christoff Iselin, 4 Bde. Basel (Johann Brandmüller ) 1726/7, zitiert als: „Basler Lexikon“. 
angräntzender Orten und Ländern Sachen betrifft/ganz umgegossen/ und um ein grosses vermehret" worden. Damit sei das größte Manko der deutschen VorgängerEnzyklopädien jetzt behoben worden. ${ }^{28} \mathrm{Im}$ Vorwort wird dann expliziert, dass diese neuen Anteile von dem in Bern lehrenden Staatsrechtler Johann Rudolf von Waldkirch stammten, der fünf Jahre zuvor eine Gründliche Einleitung zu der Eydgnossischen Bunds= und Staats=Historie veröffentlicht hatte. ${ }^{29}$

Alle diese Einlassungen sind zutreffend, doch grundsätzlich und vor allem Anderen war diese schweizerische Enzyklopädie eine Kopie oder ein unautorisierter Nachdruck (Raubdruck) des „Leipziger Lexikons“, was sich im direkten Vergleich der Lemmata mit dem „Basler Lexikon“ leicht erweisen lässt. Titel und Titelblätter des „Leipziger“ und des „Basler Lexikons“ sehen einander nicht nur zum Verwechseln ähnlich, sondern der Vorsatz „Neu vermehrt“ erweckt darüber hinaus auch den Eindruck, als handele es sich hier um eine weitere Auflage des bereits eingeführten und kurz zuvor (1722) in zweiter Auflage erschienenen Leipziger „Modells“. Die beiden um die deutschsprachige Leserschaft konkurrierenden Verleger Fritsch und Brandmüller gerieten darüber in ernsthafte Streitigkeiten, die sie mangels juristischer Mittel in Gestalt ausufernder Polemik über die Vorworte der jeweiligen Neuauflagen führten. ${ }^{30}$ Der Baseler Verleger wehrte wütend, aber unberechtigt alle Plagiatsvorwürfe ab und wies zusätzlich den unfreundlichen Hinweis zurück, sein Schweizerdeutsch sei grammatisch nicht korrekt: Die eingebildete Redaktion des deutschen Lexikons sei des Französischen nicht einmal so weit mächtig, dass man chamois und chameau auseinanderhalten könne. Im Lexikonartikel „Schweiz“ sei deshalb zu lesen, daß es dort in den Bergen „eine große Menge an Hirschen, Bären und Kamelen“ gäbe. ${ }^{31}$ Dieser peinliche Irrtum wurde in späteren Auflagen des „Leipziger Lexikons" stillschweigend berichtigt. Überhaupt wären die corrigenda-Listen beider enzyklopädischer Werke (und die der anderen genannten) eine Fundgrube für ,falsches' und ,richtiges‘ Wissen.

\section{Fazit}

Wenden wir uns an dieser Stelle der Frage zu, wo und wie die Dreiheit „Modell“, „Imitation“ und „Kopie“ den Transfer von Wissen beeinflusst und befördert. Bei den nicht-lateinischen, alphabetischen und von dem französischen Modell des

28. Zitate aus Titel und Einleitung (S. 3) des Neu vermehrten ... Lexicon s[...], Bd. 1.

29. Joh[ann] Rudolff von Waldkirch [(1678-1757)], Gründliche Einleitung zu der Eydgnossischen Bunds $=$ und Staats=Historie/ Vorstellend Den alten und neuen Zustand der Schweitz und der Eydgnossen [...], 2 Theile, Basel 1721.

30. S. Ina Ulrike Paul, Niemals ohne Gewähr. Über die Quellen nationaler Eigen- und Fremdbilder in europäischen Enzyklopädien und Universallexika, in Paul Michel/ Madeleine Herren/Martin Rüesch (Hrsg.), Allgemeinwissen und Gesellschaft, Aachen 2007, S. 195- 225; als (e-book 2006: http://www.enzyklopaedie.ch/kongress/publikation.htm)

31. Artikel Schweitz / Schweitzerland, in "Leipziger Lexikon“, 1. Aufl. Leipzig 1709, Bd. $3 / 4$ (in einem), S. 401f. - Kritik an den falschen „cameelen“: „Basler Lexikon“, 3. Teil (1726), Vorrede, S. 12. 
Moréri ausgehenden Enzyklopädien in den Landessprachen Europas lässt sich von einer ,Verflechtungsgeschichte der jeweiligen epigonalen Werke mit ihren modellhaften Vorläufern sprechen. Das war aber kein Wunder der Aufklärung, sondern das rationale Denken von Geschäftsleuten des 18. Jahrhunderts oder eben ,marktanalytische' Geschäftspolitik im Verlagswesen, in der sich der Ideen- und der Buchmarkt vereinten: Als Modell gab es zwei enzyklopädische ,Bestseller. Nach den Verkaufserfolgen der ersten modernen enzyklopädischen Wörterbücher in Frankreich wussten die Verleger, dass man mit einer Enzyklopädie „glänzende[...] Geschäfte“ machen konnte, und wollten diesen Erfolg imitieren und kopieren. ${ }^{32}$ In dem „Basler Lexikon“ liegt eine schweizerische Kopie der aus dem Französischen ins Deutsche übersetzten Moréri-Adaptation vor, die das Modell „Moréri“ imitierte. Der Basler Verleger griff damit nicht nur auf den um „Deutsches“ und um die Sicht auf die protestantische Konfession veränderten Lemmata zurück, sondern auch auf die Literaturbasis der „Leipziger Lexikons“, die aus drei Moréri-Revisionen in unterschiedlichen Landessprachen (unter Anreicherung der englischen und irischen Artikel und der konfessionellen Veränderungen) sowie Bayles skeptizistischem Wörterbuch und einem orientalistischenWerkbestanden.DamitwarenallehierbehandeltenÜbersetzungen bereits existierender Enzyklopädien in verschiedene Landessprachen von der ausschnittsweisen Revision (Fehlerbereinigung, Ergänzung, Aktualisierung) und einer landesspezifischen inhaltlichen Modifikationen der Inhalte geprägt. Das Modell aller landessprachlichen Enzyklopädien war und blieb Moréri, Kopien und Imitationen allerdings betonten die jeweilige Verbundenheit des neuen Werkes mit der anwachsenden Menge anerkannter Vorgänger-Wörterbücher. Hätte man eine beliebige Moréri-Adaptation - die niederländische oder die schweizerische etwa - in die französische Sprache rückübersetzt, so wären weder Kopien noch Imitationen dem Modell mehr als entfernt ähnlich gewesen - Adaptation, Revision und Anreicherung der Lemmata hatten neue Bücher und neue Wissenswirklichkeiten erzeugt.

32. Robert Darnton, Glänzende Geschäfte. Die Verbreitung von Diderots Encyclopédie oder: Wie verkauft man Wissen mit Gewinn? Berlin, Wagenbach, 1979. 